\title{
On the invariance and general cohomology comparison theorems
}

\author{
Alin Stancu
}

Received: 19 May 2011 / Accepted: 12 September 2012 / Published online: 5 October 2012 (C) Tbilisi Centre for Mathematical Sciences 2012

\begin{abstract}
The Invariance Theorem of Gerstenhaber and Schack states that if $\mathbb{A}$ is a diagram of algebras then the subdivision functor induces a natural isomorphism between the Yoneda cohomologies of the category $\mathbb{A}$-mod and its subdivided category $\mathbb{A}^{\prime}$-mod. In this paper we generalize this result and show that the subdivision functor is a full and faithful functor between two suitable derived categories of $\mathbb{A}$-mod and $\mathbb{A}^{\prime}$-mod. This result combined with our work in Stancu (Hochschild cohomology and derived categories, PhD thesis, SUNY, Buffalo, 2006; J Homotopy Relat Struct 6(1):39-63, 2011), on the Special Cohomology Comparison Theorem, constitutes a generalization of Gerstenhaber and Schack's General Cohomology Comparison Theorem (GCCT).
\end{abstract}

Keywords Derived category · Cohomology comparison theorem ·

Yoneda cohomology $\cdot$ Hochschild cohomology

Mathematics Subject Classification (2000) Primary 18; Secondary 16

\section{Some background}

The General Cohomology Comparison Theorem of Gerstenhaber and Schack (GCCT) asserts that the cohomology (and deformation theory) of a presheaf of unital associative algebras over a small category (called a diagram of algebras) is canonically isomorphic with the cohomology (and deformation theory) of a single algebra. The first step in

Communicated by Jim Stasheff.

A. Stancu $(\bowtie)$

Department of Mathematics, Columbus State University, Columbus, GA 31907, USA

e-mail: stancu_alin1@columbusstate.edu 
proving the GCCT is the Special Cohomology Comparison Theorem (SCCT) which deals with the case in which the small category is a poset (see [3]). A second key ingredient is the Invariance Theorem (IT), also due to Gerstenhaber and Schack. This theorem states that the barycentric subdivision of the underlying category does not change the cohomology of the diagram. Finally, since the second subdivision of a small category is a poset the SCCT and IT imply the GCCH. The IT is stated in [2], but without a proof. The complete proof exists but it is not generally available because it was not published.

In [6] and [7] we proved a generalization of the SCCT. We showed there that a certain functor, denoted "!", between the categories of bimodules over a diagram and of bimodules over the algebra associated to the diagram, extends to a full and faithful functor between two suitable relative derived categories.

By taking a very interesting, but different, approach Lowen and Van Den Bergh also proved that the functor "!" is full and faithful, thus generalizing the SCCT (see [4]). The authors indicate in that paper that their methods may be used to give new insights on the GCCT but that this will be covered elsewhere.

The purpose of this work is to use our techniques developed in [6] and [7] in order to provide a new insight on the GCCT. In the process we prove a generalization of IT (Theorem 4.9). Then we show that the GCCT is a consequence of the fact that, at level of derived categories, the subdivision functor and "!" are full and faithful (Theorem 5.3).

\section{Preliminaries}

Let $k$ be a commutative ring and $\mathcal{C}$ be a small category, i.e a category whose class of objects is a set. The objects of $\mathcal{C}$ will be denoted $h, i, j$, etc. and the maps will be $u, v, w$, etc. We will write $\operatorname{Hom}_{\mathcal{C}}(i, j)$ for the set of maps $i \rightarrow j$ and denote the domain and the codomain of a map $v$ by $d v$ and $c v$, respectively. A delta is a small category in which the only endomorphisms are identity maps and the condition $\operatorname{Hom}_{\mathcal{C}}(i, j) \neq \emptyset$ implies $\operatorname{Hom}_{\mathcal{C}}(j, i)=\emptyset$, for all $i \neq j$ in $\mathcal{C}$.

When $A$ is a $k$-algebra and $M$ any $A$ bimodule we assume $M$ to be symmetric over $k$. (i.e. $a x=x a$ for all $x \in M$ and $a \in k$.) The category of associative unital $k$-algebras will be denoted by $k$-alg. A presheaf of $k$-algebras over $\mathcal{C}$ is a contravariant functor $\mathbb{A}: \mathcal{C}^{o p} \longrightarrow k$-alg. We will denote $\mathbb{A}(i)$ by $\mathbb{A}^{i}$ and write $\varphi^{v}=\varphi_{\mathbb{A}}^{v}: \mathbb{A}^{c v} \rightarrow \mathbb{A}^{d v}$ for $\mathbb{A}(v)$. We will adopt the terminology of [2] and will call $\mathbb{A}$ a diagram over $\mathcal{C}$.

Let $\mathbb{A}$ be a diagram over $\mathcal{C}$ and $v \in \mathcal{C}$ be a map. The map $\varphi_{\mathbb{A}}^{v}: \mathbb{A}^{c v} \rightarrow \mathbb{A}^{d v}$ makes any $\mathbb{A}^{d v}$-module $M$ an $\mathbb{A}^{c v}$ module. The resulting module will be denoted by $|M|_{v}$. A left $\mathbb{A}$-module $\mathbb{M}$ is a presheaf (over $\mathcal{C}$ ) of abelian groups such that:

1. $\mathbb{M}^{i}$ is an $\mathbb{A}^{i}$-module.

2. If $v \in \mathcal{C}$ then $T^{v}=T_{\mathbb{M}}^{v}: \mathbb{M}^{c v} \rightarrow\left|\mathbb{M}^{d v}\right|_{v}$ is an $\mathbb{A}^{c v}$-module map.

An $\mathbb{A}$-module map $\eta: \mathbb{M} \longrightarrow \mathbb{N}$ is a natural transformation in which $\eta^{i}$ is an $\mathbb{A}^{i}$-module map $(\forall) i \in \mathcal{C}$. The category of left $\mathbb{A}$-modules will be denoted by $\mathbb{A}$-mod. It is abelian, complete, and cocomplete. (All constructions are made "objectwise".) The definitions of right modules and bimodules are similar and with the 
opposite diagram $\mathbb{A}^{o p}$ and the enveloping diagram $\mathbb{A}^{e}=\mathbb{A} \otimes_{k} \mathbb{A}^{o p}$ defined in the obvious way we have that the category of right $\mathbb{A}$-modules, mod- $\mathbb{A}$ and that of $\mathbb{A}$-bimodules, $\mathbb{A}$-bimod, are isomorphic to $\mathbb{A}^{o p}$-mod and $\mathbb{A}^{e}$-mod.

If $f: \mathcal{D} \rightarrow \mathcal{C}$ is a covariant functor between small categories then every diagram $\mathbb{A}: \mathcal{C}^{o p} \rightarrow k$-alg over $\mathcal{C}$ defines a diagram $f^{*} \mathbb{A}: \mathcal{D}^{o p} \rightarrow k$-alg over $\mathcal{D}$ by setting $\left(f^{*} \mathbb{A}\right)^{\sigma}=\mathbb{A}^{f \sigma}$ and $\varphi_{f * \mathbb{A}}^{v}=\varphi_{\mathbb{A}}^{f v}$. Moreover, the functor $f$ induces a functor $f^{*}: \mathbb{A}$-mod $\rightarrow\left(f^{*} \mathbb{A}\right)$-mod by setting $\left(f^{*} \mathbb{N}\right)^{\sigma}=\mathbb{N}^{f \sigma}, T_{f * \mathbb{N}}^{v}=T_{\mathbb{N}}^{f v}$, and $\left(f^{*} \eta\right)^{\sigma}=\eta^{f \sigma}$. Note that $f^{*}$ is an exact embedding.

In [3] Gerstenhaber and Schack proved that the functor $f^{*}$ has both a left and a right adjoint. Because we will use the left adjoint to prove a generalization of their Invariance Theorem we include Gerstenhaber and Schack's description.

Let $f: \mathcal{D} \rightarrow \mathcal{C}$ be a functor as above and $i$ an object in $\mathcal{C}$. Then the comma category $\mathbf{i} / \mathbf{f}$ is the category whose objects are the $\mathcal{C}$-maps $i \stackrel{w}{\longrightarrow} f \sigma$ where $\sigma \in \mathcal{D}$. Such an object will be denoted by $(w, \sigma)$. A map $(u, \tau) \rightarrow(w, \sigma)$ in $\mathbf{i} / \mathbf{f}$ is simply a $\mathcal{D}$-map $v: \tau \rightarrow \sigma$ such that $u(f v)=w$. A $\mathcal{C}$-map $h \stackrel{v}{\longrightarrow} i$ induces a functor $\mathbf{i} / \mathbf{f} \rightarrow \mathbf{h} / \mathbf{f}$ described on objects by $(w, \sigma) \rightarrow(v w, \sigma)$.

If $v \in \mathcal{C}$ then, using the map $\varphi^{v}: \mathbb{A}^{c v} \rightarrow \mathbb{A}^{d v}$, we may view $\mathbb{A}^{d v}$ as a left $\mathbb{A}^{d v}$, right $\mathbb{A}^{c v}$-module. This implies that if $M$ is any left $\mathbb{A}^{c v}$-module then $\mathbb{A}^{d v} \otimes_{\mathbb{A}^{c v}} M$ is a left $\mathbb{A}^{d v}$ module. To make the role of $v$ explicit we denote this module by $\mathbb{A}^{d v} \otimes_{v} M$. For $a \in \mathbb{A}^{d v}, b \in \mathbb{A}^{c v}$ and $m \in M$, we have $a \otimes b m=a\left(\varphi^{v} b\right) \otimes m$.

The left adjoint of $f^{*}$ is denoted by $f !:\left(f^{*} \mathbb{A}\right)$-mod $\rightarrow \mathbb{A}$-mod and defined as follows. Let $\mathbb{N}$ be an $\left(f^{*} \mathbb{A}\right)$-module. For each $i \in \mathcal{C}$ and each $(u, \tau) \stackrel{v}{\longrightarrow}(w, \sigma)$ in $\mathbf{i} / \mathbf{f}$ the map $I d \otimes T_{\mathbb{N}}^{v}: \mathbb{A}^{i} \otimes_{w} \mathbb{N}^{\sigma} \rightarrow \mathbb{A}^{i} \otimes_{u} \mathbb{N}^{\tau}$ is $\mathbb{A}^{i}$-linear. The collection of all these maps defines a diagram of $\mathbb{A}^{i}$-modules over $\mathbf{i} / \mathbf{f}$ by setting

$$
\left(f_{!} \mathbb{N}\right)^{i}=\operatorname{colim}_{(w, \sigma) \in \mathbf{i} / \mathbf{f}} \mathbb{A}^{i} \otimes_{w} \mathbb{N}^{\sigma} .
$$

Also, for each $v \in \operatorname{Hom}_{\mathcal{C}}(h, i)$ and $(w, \sigma) \in \mathbf{i} / \mathbf{f}$ there is an $\mathbb{A}^{i}$-module map $\varphi_{\mathbb{A}}^{v} \otimes I d$ : $\mathbb{A}^{i} \otimes_{w} \mathbb{N}^{\sigma} \rightarrow \mathbb{A}^{h} \otimes_{v w} \mathbb{N}^{\sigma}$. The universal property of colimits implies that the functor $\mathbf{i} / \mathbf{f} \rightarrow \mathbf{h} / \mathbf{f}:(w, \sigma) \rightarrow(v w, \sigma)$ induces an $\mathbb{A}^{i}$-module map $T^{v}:\left(f_{!} \mathbb{N}\right)^{i} \rightarrow(f ! \mathbb{N})^{h}$ and that $T^{u} T^{v}=T^{u v}$. This implies that these modules and maps form an $\mathbb{A}$-module $f_{!} \mathbb{N}$. By the universality property of colimits each $f^{*} \mathbb{A}$-module map $\mathbb{N} \rightarrow \mathbb{M}$ induces an $\mathbb{A}$-module map $f_{!} \mathbb{N} \rightarrow f_{!} \mathbb{M}$, so $f_{!}$is a functor. For a proof that $f_{!}$is a left adjoint of $f^{*}$ the reader could see [2].

Yoneda cohomology of the category $\mathbb{A}$-mod is closely related to the notion of "allowable" map. These maps will also play an important role in defining the relative derived category of $\mathbb{A}$-mod, so we remind the reader of their definition. A map $\eta$ : $\mathbb{M} \longrightarrow \mathbb{N}$ is called allowable if $(\forall) i \in \mathcal{C}$ the map $\eta^{i}: \mathbb{M}^{i} \longrightarrow \mathbb{N}^{i}$ admits a $k$ module splitting map $k^{i}: \mathbb{N}^{i} \longrightarrow \mathbb{M}^{i}$ satisfying $\eta^{i} k^{i} \eta^{i}=\eta^{i}$. We do not require the splitting maps $k^{i}$ to be natural. An $\mathbb{A}$-module $\mathbb{P}$ is called relative projective if for every allowable epimorphism $\mathbb{M} \longrightarrow \mathbb{N}$ the induced map $\operatorname{Hom}_{\mathbb{A}}(\mathbb{P}, \mathbb{M}) \longrightarrow \operatorname{Hom}_{\mathbb{A}}(\mathbb{P}, \mathbb{N})$ is an epimorphism of sets.

A relative projective allowable resolution of an $\mathbb{A}$-module $\mathbb{M}$ is an exact sequence $\cdots \longrightarrow \mathbb{P}_{n} \cdots \longrightarrow \mathbb{P}_{1} \longrightarrow \mathbb{P}_{0} \longrightarrow \mathbb{M} \longrightarrow 0$ in which all $\mathbb{P}_{n}$ are relative projective 
$\mathbb{A}$-modules and all maps are allowable. The category $\mathbb{A}$-mod has enough relative projective modules and each module has a relative projective allowable resolution. Moreover, there is a functorial way of getting this type of resolutions. The construction of such a resolution is due to Gerstenhaber and Schack (see [2]). They called it the generalized simplicial bar (GSB) resolution.

\section{The subdivision of a category}

Let $\mathcal{C}$ be a small category. If [p] is the linearly ordered set $\{0<\cdots<p\}$ viewed as a category then a $p$-simplex is a covariant functor $\sigma:[\mathbf{p}] \rightarrow \mathcal{C}$. In this case we say that the dimension of $\sigma$ is $p$ and we write $\operatorname{dim} \sigma=p$. A functor $f:[\mathbf{p}] \rightarrow[\mathbf{q}]$ is called monotone if and only if $i<j$ implies $f i<f j$.

Every small category $\mathcal{C}$ has a subdivision $\mathcal{C}^{\prime}$ which is again a category. The objects of the subdivision $\mathcal{C}^{\prime}$ are the simplices of the category $\mathcal{C}$. To define the maps let $\tau$ and $\sigma$ be objects in $\mathcal{C}^{\prime}$ such that $\operatorname{dim} \tau=p$ and $\operatorname{dim} \sigma=q$. A map $\tau \rightarrow \sigma$ in $\mathcal{C}^{\prime}$ is a triple $[\tau, \sigma, v]$ where $\tau$ is the domain, $\sigma$ the codomain, and $v$ a map in $\mathcal{C}$ such that there exists a monotone functor $f:[\mathbf{q}] \rightarrow[\mathbf{p}]$ such that the triangle

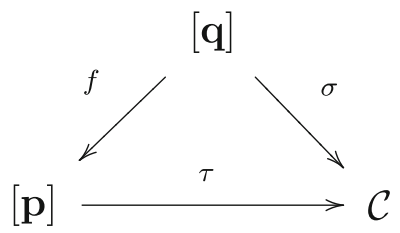

commutes and $v=\tau^{0, f 0}=\tau(0 \rightarrow f 0)=\tau(0) \rightarrow \sigma(0)$.

The definition of the barycentric subdivision of a small category of [2] is so commonly accepted that it is not attributed to anyone. It is an important construction and to my knowledge it is due to Schack.

Note that if $\operatorname{dim} \tau<\operatorname{dim} \sigma$, then there are no maps $\tau \rightarrow \sigma$. The composition is written in diagrammatic order and is given by $[\tau, \sigma, u] \circ[\sigma, \omega, v]=[\tau, \omega, u v]$. It is not hard to see that the following proposition is true.

Proposition 3.1 If $\mathcal{C}$ is a small category then $\mathcal{C}^{\prime}$ is a delta. In addition, if $\mathcal{C}$ is a delta then $\mathcal{C}^{\prime}$ is a poset. In particular, the second subdivision $\mathcal{C}^{\prime \prime}$ of a small category $\mathcal{C}$ is a poset.

The subdivision of a small category $\mathcal{C}$ induces a functor $\mathbf{d}: \mathcal{C}^{\prime} \rightarrow \mathcal{C}$ defined on objects by $\mathbf{d} \tau=\tau(0)$ and on maps by $\mathbf{d}[\tau, \sigma, v]=v$. Each functor $f: \mathcal{D} \rightarrow \mathcal{C}$ induces a functor $f^{\prime}: \mathcal{D}^{\prime} \rightarrow \mathcal{C}^{\prime}$ by taking $f^{\prime}([\mathbf{p}] \stackrel{\tau}{\longrightarrow} \mathcal{D})$ to be the composite $[\mathbf{p}] \stackrel{\tau}{\longrightarrow} \mathcal{D} \stackrel{f}{\longrightarrow} \mathcal{C}$ and $f^{\prime}([\tau, \sigma, v])=\left[f^{\prime}(\tau), f^{\prime}(\sigma), f(v)\right]$. The subdivision is a functor from the category of small categories to itself and $\mathbf{d}$ is a natural transformation from this functor to the identity functor.

In [2] Gerstenhaber and Schack used the functor $\mathbf{d}: \mathcal{C}^{\prime} \rightarrow \mathcal{C}$ to "subdivide" any diagram $\mathbb{A}$ over $\mathcal{C}$ obtaining a new diagram over $\mathcal{C}^{\prime}, \mathbf{d}^{*} \mathbb{A}=\mathbb{A}^{\prime}$, as follows:

$$
\left(\mathbb{A}^{\prime}\right)^{\tau}=\mathbb{A}^{d \tau} \text { and } \varphi_{\mathbb{A}^{\prime}}^{[\tau, \sigma, v]}=\varphi_{\mathbb{A}}^{d[\tau, \sigma, v]}=\varphi_{\mathbb{A}}^{v}
$$


It follows from the general case described in the previous section that the induced functor $\mathbf{d}^{*}: \mathbb{A}$-mod $\rightarrow \mathbb{A}^{\prime}$-mod preserves allowability and has a left adjoint $\mathbf{d}_{\text {! }}$ which preserves relative projectives.

It is not hard to see that $\mathbf{d}^{*}$ is full and faithful, so we have $\mathbf{d}_{!} \mathbb{M}^{\prime} \cong \mathbb{M}$. In fact, Gerstenhaber and Schack proved the more general result:

Theorem 3.2 (The Invariance Theorem) The natural transformation induced by $\mathbf{d}^{*}$ induces and isomorphism

$$
\mathbf{E x t}_{\mathbb{A}, k}^{\bullet}(-,-) \rightarrow \mathbf{E x t}_{\mathbb{A}^{\prime}, k}^{\bullet}\left((-)^{\prime},(-)^{\prime}\right) .
$$

Our effort in the next section is to generalize this theorem. We show that in a certain derived category, where we may view the relative Yoneda cohomology as homomorphism groups, the extension of $\mathbf{d}^{*}$ is full and faithful. This result combined with our work in [6] and [7], on Gerstenhaber and Schack's Special Cohomology Comparison Theorem, constitutes a generalization of their General Cohomology Comparison Theorem.

A very important ingredient in our work is the following theorem of Gerstenhaber and Schack.

Theorem 3.3 Let $\mathbb{N}$ be an $\mathbb{A}$-module. There is a relative projective allowable resolution $\mathbb{N}_{\bullet} \rightarrow \mathbb{N}^{\prime}$ of the subdivided module $\mathbb{N}^{\prime}$ in $\mathbb{A}^{\prime}-\bmod$ such that $\mathbf{d} ! \mathbb{P} \bullet \rightarrow \mathbb{N}$ is a relative projective allowable resolution of $\mathbb{N}$ in $\mathbb{A}$-mod.

They obtained the resolution $\mathbb{P}_{\bullet} \rightarrow \mathbb{N}^{\prime}$ by "spreading out" the GSB resolution of $\mathbb{N}$ over $\mathcal{C}^{\prime}$. The resolution $\mathbf{d}_{!} \mathbb{P} \bullet \rightarrow \mathbb{N}$ is exactly the GSB resolution of $\mathbb{N}$. One might think that every $\mathbb{A}^{\prime}$-module $\mathbb{N}$ has a relative projective allowable resolution $\mathbb{P} \bullet \rightarrow \mathbb{N}$ for which $\mathbf{d}_{!} \mathbb{P} \bullet \rightarrow \mathbf{d} ! \mathbb{N}$ is also a relative projective allowable resolution, but in general this is not true. When $k$ is a field this is equivalent to the exactness of $\mathbf{d}_{\text {! }}$, so it can't hold in general.

\section{The invariance theorem}

We construct now the relative derived category $\mathcal{D}_{k}^{-}(\mathbb{A}$-mod) in which the Yoneda cohomology of $\mathbb{A}$-mod can be identified with the homomorphism groups.

This construction is inspired by the ideas of [6] and [7] where we defined a relative derived category of $\mathbb{A}$-bimod and showed that this category is an appropriate setting to generalize the Special Cohomology Comparison Theorem (SCCT) of presheaves over posets. Because SCCT is a bimodule-only statement our approach required the use of a relative derived category of bimodules.

The Invariance Theorem is a statement about one-sided modules over an arbitrary diagram $\mathbb{A}$ so an appropriate setting to extend it is to use a derived category of $\mathbb{A}$-mod. It is not hard to see that the one-sided context extends naturally from the bimodule scenario of [6] and [7] and that many results are independent of the particularity of bimodules. Those results will be stated in the one-sided case without a proof. In hindsight, a more natural approach in [6] and [7] could have been to define the relative 
derived category of $\mathbb{A}$-mod and then to use it for bimodules via the enveloping diagram $\mathbb{A}^{e}=\mathbb{A} \otimes_{k} \mathbb{A}^{o p}$.

Let $\mathbb{A}$ be a diagram over $\mathcal{C}$ and let $\mathrm{Kom}^{-}(\mathbb{A}$-mod) be the category of bounded to the right complexes of $\mathbb{A}$-modules

$$
\mathbb{M}_{\bullet}:=\cdots \mathbb{M}_{n} \longrightarrow \cdots \quad \cdots \longrightarrow \mathbb{M}_{1} \longrightarrow \mathbb{M}_{0} \longrightarrow 0
$$

A map between two complexes $\mathbb{M}_{\bullet}$ and $\mathbb{N}_{\bullet}$ is a collection of maps $f=\left(f_{i}\right)$ : $\mathbb{M}_{i} \rightarrow \mathbb{N}_{i}$, one for each positive integer $i$, which commute with the differentials of $\mathbb{M}_{\bullet}$ and $\mathbb{N}_{\bullet}$. We do not require the maps defining the complexes or the maps between complexes to be $k$-split. We denote the homotopic category of $\mathrm{Kom}^{-}(\mathbb{A}$-mod) by $\mathcal{K}^{-}(\mathbb{A}-$ mod $)$.

Definition 4.1 A map $\mathbb{M}_{\bullet} \stackrel{f}{\longrightarrow} \mathbb{N}_{\bullet}$ in $\mathrm{Kom}^{-}(\mathbb{A}$-mod) is a relative quasi-isomorphism if the maps of complexes of $\mathbb{A}^{i}$-modules $\mathbb{M}_{\bullet}^{i} \stackrel{f^{i}}{\longrightarrow} \mathbb{N}_{\bullet}^{i}$ have contractible cones, when considered as complexes of $k$-modules, for all $i \in \mathcal{C}$.

The word "relative" is a reminder to the reader that Yoneda cohomology is a relative theory, since $k$ is a commutative ring that is not necessarily a field.

The following proposition characterizes relative quasi-isomorphisms and is an ingredient in proving that the class of these maps is localizing in $\mathcal{K}^{-}(\mathbb{A}$-mod). It was proved in [6] and [7] for $\mathbb{A}$-bimodules and the same proof works for one sided A-modules.

Proposition 4.2 Let $A$ be any $k$-algebra and $f: M_{\bullet} \longrightarrow N_{\bullet}$ a map of complexes of $A$-modules in $\mathrm{Kom}^{-}(A$-mod). Then, $f$ is a relative quasi-isomorphism if and only if there exists $\gamma: N_{\bullet} \longrightarrow M_{\bullet}$ a map of complexes of $k$-modules such that $f \gamma \sim i d_{N_{\bullet}}$ and $\gamma f \sim i d_{M_{\bullet}}$ in $\mathrm{Kom}^{-}(k$-mod), where ' $\sim$ 'stands for homotopy equivalence.

Proof See [7], proposition 3.2.

As a corollary, we note that if any two of $f, g$ or $f g$ are relative quasi-isomorphisms then so is the third. This can be uses to prove the following result.

Proposition 4.3 The class of relative quasi-isomorphisms in the homotopic category $\mathcal{K}^{-}(\mathbb{A}$-mod $)$ is localizing.

Proof See [7], proposition 3.3.

We define now the relative derived category of $\mathbb{A}$-mod.

Definition 4.4 $\mathcal{D}_{k}^{-}(\mathbb{A}$-mod $):=\mathcal{K}^{-}(\mathbb{A}$-mod $)\left(\Sigma^{-1}\right)$, where $\mathcal{K}^{-}$is the homotopy category and $\Sigma$ is the class of relative quasi-isomorphisms in $\mathcal{K}^{-}(\mathbb{A}$-mod).

Because $\Sigma$ is localizing we may regard the morphisms in $\mathcal{D}_{k}^{-}(\mathbb{A}-\mathbf{m o d})$ as equivalence classes of diagrams

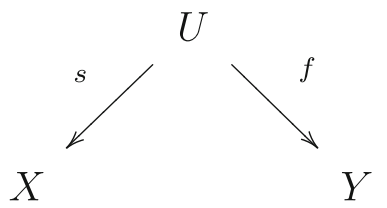


The maps $s$ and $f$ are morphisms in the homotopy category with $s \in \Sigma$. These diagrams are usually called roofs and we adopt this terminology. In addition, because $\Sigma$ is a localizing class the relative derived category is triangulated.

Proposition 4.5 Let $\mathbb{P} \bullet$ be a complex of relative projective $\mathbb{A}$-modules and $\mathbb{M}_{\bullet} \stackrel{f}{\longrightarrow} \mathbb{N}$. a relative quasi-isomorphism. Then

a) $\operatorname{Mor}_{\mathcal{K}}-(\mathbb{A}$-mod $)\left(\mathbb{P}_{\bullet}, \mathcal{C}(f)_{\bullet}\right)=0$.

b) The canonical map induced by $f$

$$
\operatorname{Mor}_{\mathcal{K}^{-}(\mathbb{A} \text {-mod })}\left(\mathbb{P}_{\bullet}, \mathbb{M}_{\bullet}\right) \stackrel{\mathbf{f}}{\longrightarrow} \operatorname{Mor}_{\mathcal{K}^{-}(\mathbb{A}-\text { mod })}\left(\mathbb{P}_{\bullet}, \mathbb{N}_{\bullet}\right)
$$

is onto.

c) The canonical map

$$
\operatorname{Mor}_{\mathcal{K}^{-}(\mathbb{A}-\text { mod })}\left(\mathbb{P}_{\bullet}, \mathbb{Q}_{\bullet}\right) \stackrel{\text { can }}{\longrightarrow} \operatorname{Mor}_{\mathcal{D}_{k}^{-}(\mathbb{A}-\bmod )}\left(\mathbb{P}_{\bullet}, \mathbb{Q}_{\bullet}\right)
$$

is an isomorphism for every $\mathbb{Q}_{\bullet} \in \mathrm{Kom}^{-}(\mathbb{A}$-mod $)$.

Proof See [7], lemma 3.5 and proposition 3.6.

The proposition helps us establish the connection between the relative Yoneda cohomology of $\mathbb{A}-\bmod$ and $\mathcal{D}_{k}^{-}(\mathbb{A}$-mod $)$.

Theorem 4.6 $\operatorname{Ext}_{\mathbb{A}, \mathbf{k}}^{i}(\mathbb{M}, \mathbb{N}) \simeq \operatorname{Mor}_{\mathcal{D}_{k}^{-}(\mathbb{A}-\bmod )}\left(\mathbb{M}_{\bullet}, \mathbb{N}_{\bullet}[i]\right)$.

Proof See [7], theorem 3.9.

The next result gives sufficient conditions for the total complex of a double complex to be homotopic equivalent with its augmented column.

Proposition 4.7 Let $A$ be a k-algebra and assume that we have a double complex of A-modules

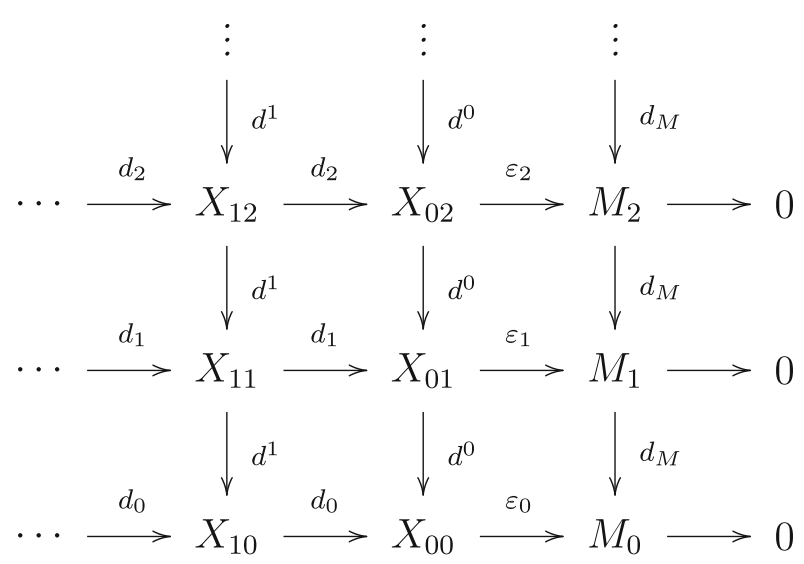


such that:

a) Each row is k-contractible. (i.e. There exist $k$-module maps $X_{(h-1) i} \stackrel{t_{i}^{h}}{\longrightarrow} X_{h i}$ such that $d_{i} t_{i}^{h+1}+t_{i}^{h} d_{i}=i d_{X_{h i}}$.)

b) The following diagrams are commutative:

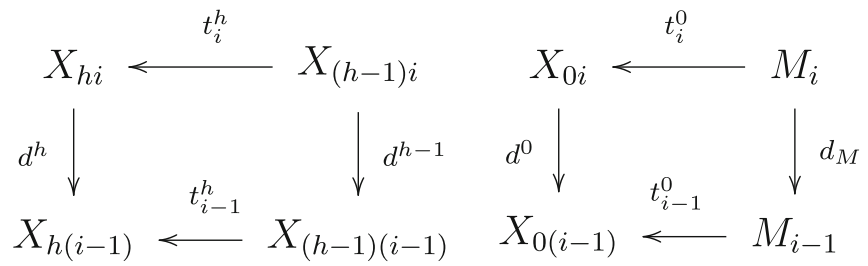

for all $h, i \geq 0$, Then

1. $M_{\bullet} \stackrel{t_{\bullet}^{0}}{\longrightarrow}\left(T\right.$ ot $\left.X_{\bullet \bullet}\right)$ and $\left(T\right.$ ot $\left.X_{\bullet \bullet}\right) \stackrel{\varepsilon_{\bullet}}{\longrightarrow} M_{\bullet}$ are maps of complexes of $k$-modules, where $\varepsilon_{i}=0$ on $X_{j h}, j+h=i$ if $j>0$.

2. $\varepsilon_{\bullet} t_{\bullet}^{0}=i d_{M_{\bullet}}$ and $t_{\bullet}^{0} \varepsilon_{\bullet} \sim i d_{T o t} X_{\bullet}$ in $\mathrm{Kom}^{-}(k-\bmod )$, where $\sim=$ homotopy equivalence.

Proof See [7], proposition 3.7.

The proposition is a key ingredient in justifying the next theorem.

Theorem 4.8 For each $\mathbb{M}_{\bullet} \in \mathcal{D}_{k}^{-}(\mathbb{A}$-mod $)$ there exist a complex of relative projective $\mathbb{A}^{\prime}$-modules $\mathcal{U} \mathbb{M}_{\bullet} \in \mathcal{D}_{k}^{-}\left(\mathbb{A}^{\prime}\right.$-mod $)$ and a relative quasi-isomorphism $\varepsilon: \mathcal{U} \mathbb{M}_{\bullet} \rightarrow \mathbb{M}_{\bullet}^{\prime}$ in $\mathcal{D}_{k}^{-}\left(\mathbb{A}^{\prime}\right.$-mod $)$.

Proof Using Theorem 3.3, for each term $\mathbb{M}_{i}^{\prime}$ of the complex $\mathbb{M}_{\bullet}, i \geq 0$ we obtain a double complex of relative projective $\mathbb{A}^{\prime}$-modules with augmented column $\mathbb{M}^{\prime}$. The rows of this complex are resolutions obtained by "spreading out" the GSB resolution of $\mathbb{M}$ over $\mathcal{C}^{\prime}$. In addition, each such row has a contracting homotopy and for each $\tau \in \mathcal{C}^{\prime}$ we obtain a double complex of $\left(\mathbb{A}^{\prime}\right)^{\tau}$-modules which satisfies the conditions of the proposition 3.7. Thus, by taking the total complex of the double complex with augmented column $\mathbb{M}^{\prime}$, we obtain a complex of relative projective $\mathbb{A}^{\prime}$-modules, $\mathcal{U} \mathbb{M}$. and a relative quasi-isomorphism $\mathcal{U} \mathbb{M}_{\bullet} \stackrel{\varepsilon}{\longrightarrow} \mathbb{M}_{\bullet}^{\prime}$. Moreover, by applying the functor $\mathbf{d}_{\text {! }}$ to this relative quasi-isomorphism and using Theorem 3.3 in combination with the fact that $\mathbf{d}^{*}$ is full and faithful, we get that $\mathbf{d}_{!}(\varepsilon)$ is a relative quasi-isomorphism from the total complex obtained by taking the GSB resolution of each $\mathbb{M}_{i}$, to $\mathbb{M}_{\text {}}$.

Since the functor $\mathbf{d}^{*}: \mathbb{A}$-mod $\rightarrow \mathbb{A}^{\prime}$-mod is exact, $k$-linear, and preserves allowable maps, it preserves relative quasi-isomorphisms as well. Thus, it induces a $k$-linear functor $\mathbf{d}^{*}$ at the level of relative derived categories. We now prove the following generalization of the Invariance Theorem. 
Theorem 4.9 The functor $\mathbf{d}^{*}: \mathcal{D}_{k}^{-}(\mathbb{A}-\bmod ) \rightarrow \mathcal{D}_{k}^{-}\left(\mathbb{A}^{\prime}-\bmod \right)$ is full and faithful. That is,

$$
\operatorname{Mor}_{\mathcal{D}_{k}^{-}(\mathbb{A}-\mathbf{m o d})}\left(\mathbb{M}_{\bullet}, \mathbb{N}_{\bullet}\right) \stackrel{\mathbf{d}^{*}}{\longrightarrow} \operatorname{Mor}_{\mathcal{D}_{k}^{-}\left(\mathbb{A}^{\prime}-\mathbf{m o d}\right)}\left(\mathbb{M}^{\prime} \bullet, \mathbb{N}^{\prime} \bullet\right)
$$

is an isomorphism of $k$-modules for all $\mathbb{M}_{\bullet}$ and $\mathbb{N}_{\bullet}$ in $\mathcal{D}_{k}^{-}(\mathbb{A}-\bmod )$.

Proof Let

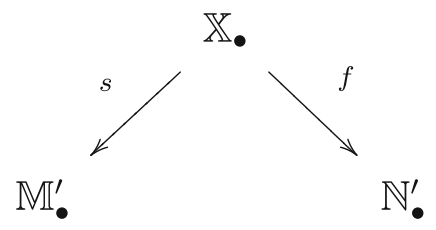

be a roof in $M o r_{\mathcal{D}_{k}^{-}\left(\mathbb{A}^{\prime}-\text { mod }\right)}\left(\mathbb{M}^{\prime} \bullet, \mathbb{N}_{\bullet}^{\prime}\right)$. Take $\mathcal{U} \mathbb{M}_{\bullet}$ and $\varepsilon$ as in the previous theorem. Since $\mathbb{X}_{\bullet} \stackrel{s}{\longrightarrow} \mathbb{M}_{\bullet}^{\prime}$ is a relative quasi-isomorphism and $\mathcal{U} \mathbb{M}_{\bullet}$ is a complex of relative projective $\mathbb{A}^{\prime}$-modules then proposition 4.5 implies that there exist $q \in \operatorname{Mor}_{\mathcal{K}^{-}(\mathbb{A} \text {-mod })}\left(\mathcal{U} \mathbb{M}_{\bullet}, \mathbb{X}_{\bullet}\right)$ such that $q s=\varepsilon$. Moreover, $q$ is a relative quasi-isomorphism because both $s$ and $\varepsilon$ are. We now have the equivalence of roofs

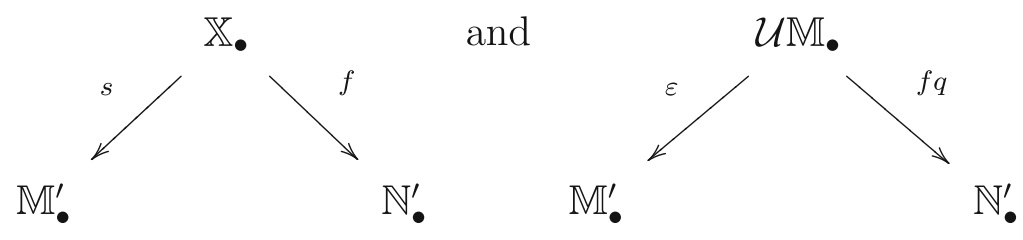

because of the following commutative diagram

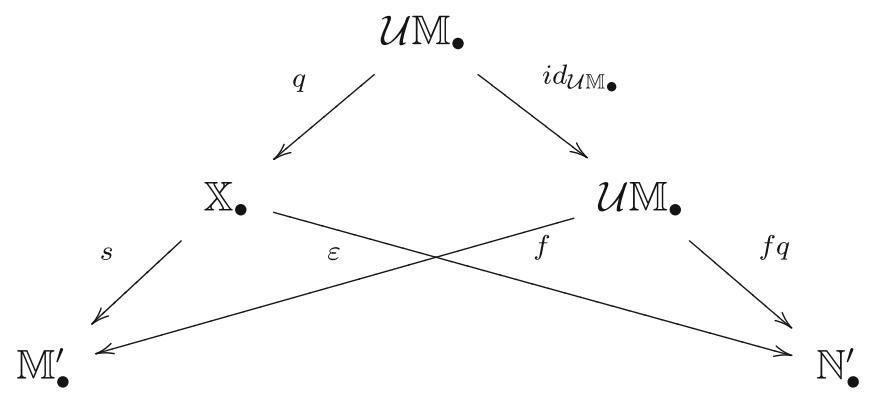

If $\varepsilon_{\mathbb{M}_{\bullet}}$ and $\varepsilon_{\mathbb{N}_{\bullet}}$ denote the maps of complexes induced by the counit of the adjunction $\mathbb{A}-\bmod \stackrel{\mathrm{d}^{*}}{\longleftarrow} \mathbb{A}^{\prime}-\bmod$, then note that they are isomorphisms since the $d_{!}$ 
functor $\mathbf{d}^{*}$ is full and faithful. In addition, we have that $\mathbf{d}_{!}(\varepsilon)$ is a relative quasiisomorphism in $\mathcal{D}_{k}^{-}(\mathbb{A}-\mathbf{m o d})$, so the roof

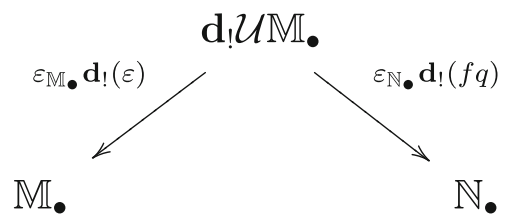

exists in this category.

We show now that the image of this roof through $\mathbf{d}^{*}$ is equivalent to

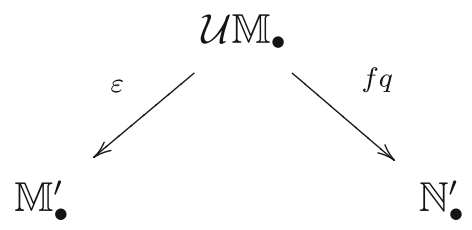

To see this note that if $\eta$ is the unit of the adjunction, naturally extended to complexes, we have $\mathbf{d}^{*}\left(\varepsilon_{\mathbb{M}_{\mathbf{0}}}\right) \eta_{\mathbf{d}^{*}\left(\mathbb{M}_{\bullet}\right)}=i d_{\mathbf{d}^{*}\left(\mathbb{M}_{\bullet}\right)}$. In addition, the functoriality of $\eta$ implies that $\left(\mathbf{d}^{*} \mathbf{d}_{!}\right)(\varepsilon) \eta_{\mathcal{U} \mathbb{M}_{\bullet}}=\eta_{\mathbf{d}^{*}\left(\mathbb{M}_{\bullet}\right)} \varepsilon$ and $\left(\mathbf{d}^{*} \mathbf{d}_{!}\right)(f q) \eta_{\mathcal{U} \mathbb{M}_{\bullet}}=\eta_{\mathbf{d}^{*}\left(\mathbb{M}_{\bullet}\right)} f q$. Thus, we have $\mathbf{d}^{*}\left(\varepsilon_{\mathbb{M}_{\bullet}}\right)\left(\mathbf{d}^{*} \mathbf{d}_{!}\right)(\varepsilon) \eta \mathcal{U} \mathbb{M}_{\bullet}=\varepsilon$. and $\mathbf{d}^{*}\left(\varepsilon_{\mathbb{N}_{\bullet}}\right)\left(\mathbf{d}^{*} \mathbf{d}_{!}\right)(f q) \eta \mathcal{U} \mathbb{M}_{\bullet}=f q$, so the following diagram is commutative and the surjectivity is proved.

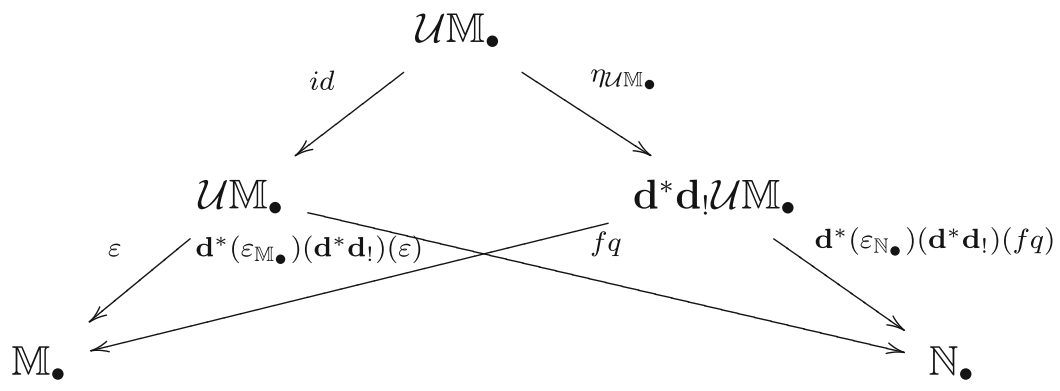

To prove that $\mathbf{d}^{*}$ is injective assume that the roofs

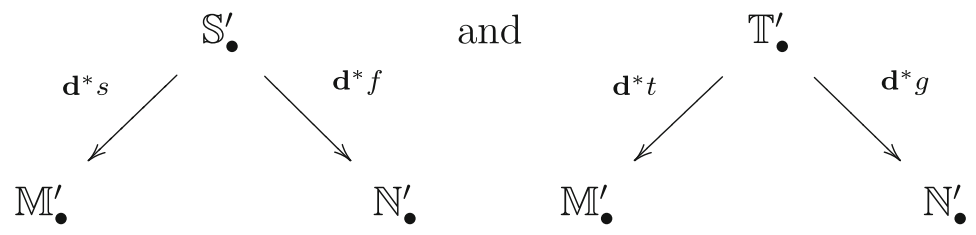


are equivalent in $\mathcal{D}_{k}^{-}\left(\mathbb{A}^{\prime}-\right.$ mod $)$. Thus, there exist a commutative diagram

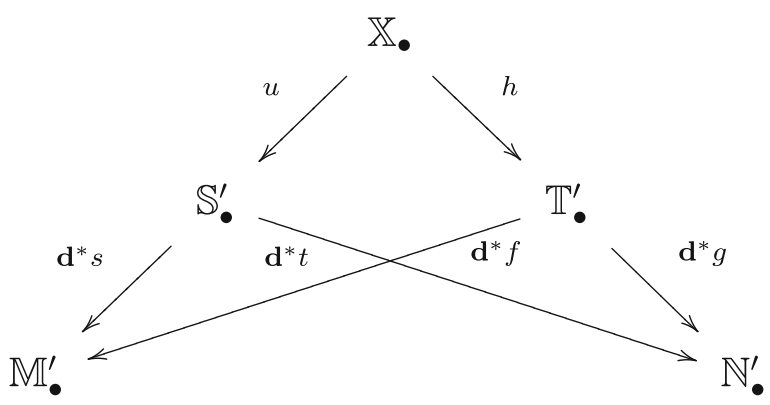

where $u$ is a relative quasi-isomorphism. We will try to replace $\mathbb{X}_{\bullet}$ with a "better" complex. For this let $\alpha: \mathcal{U} \mathbb{S}_{\bullet} \rightarrow \mathbb{S}_{\bullet}^{\prime}$ be a relative quasi-isomorphism as in the previous theorem. Because $u$ is a relative quasi-isomorphism and $\mathcal{U} \mathbb{S}$ is a complex or relative projective $\mathbb{A}^{\prime}$-modules, proposition 4.5 implies that $\operatorname{Mor}_{\mathcal{K}^{-}\left(\mathbb{A}^{\prime} \text {-mod }\right)}\left(\mathcal{U} \mathbb{S}_{\bullet}, \mathcal{C}(u) \bullet\right)=0$, so there exists a map $\beta: \mathcal{U} \mathbb{S}_{\bullet} \rightarrow \mathbb{X}_{\bullet}$ such that $u \beta=\alpha$. Moreover, since $\alpha$ and $u$ are relative quasi-isomorphisms, so is $\beta$. This implies the commutativity of the roof

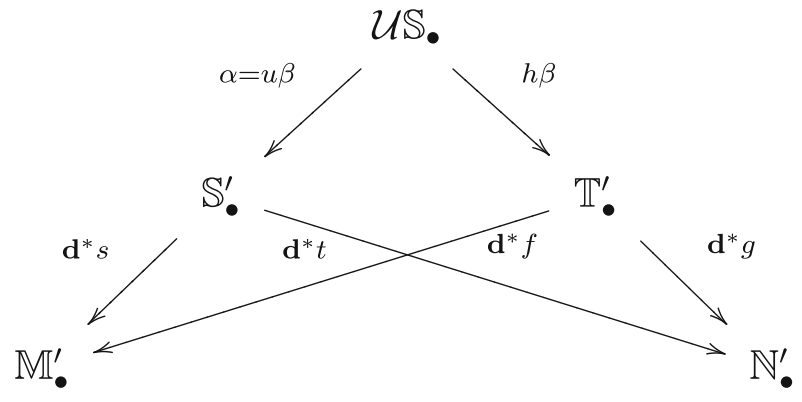

Applying the functor $\mathbf{d}_{\text {! }}$ we get the commutative diagram

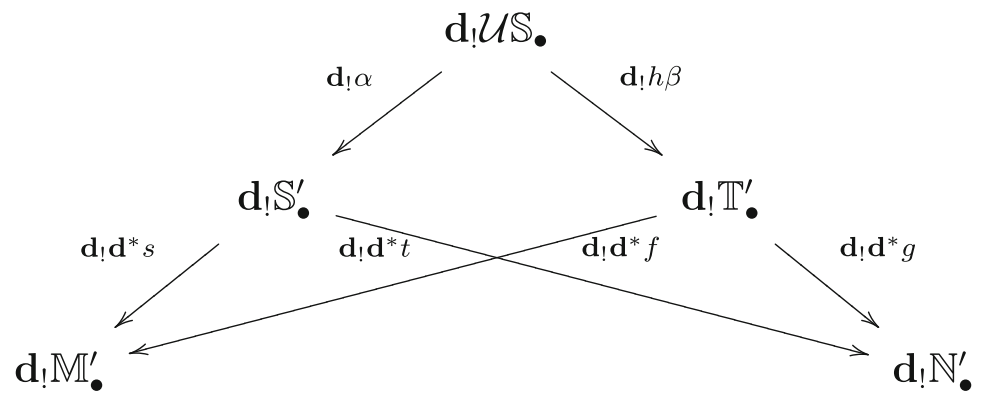

Because $\mathbf{d}_{!}(\alpha), \varepsilon_{\mathbb{M}}$. and $\mathbf{d}_{!} \mathbf{d}^{*}(s)$ are relative quasi-isomorphisms in $\mathcal{D}_{k}^{-}(\mathbb{A}$-mod) we get that the last diagram is an equivalence of roofs in $\mathcal{D}_{k}^{-}(\mathbb{A}$-mod $)$. Since $\varepsilon_{\mathbb{R}}: \mathbf{d} ! \mathbb{R}_{\bullet}^{\prime} \rightarrow$ $\mathbb{R}_{\bullet}$ is an isomorphism for all $\mathbb{R}_{\bullet} \in \mathcal{D}_{k}^{-}(\mathbb{A}$-mod) we get that the roofs 


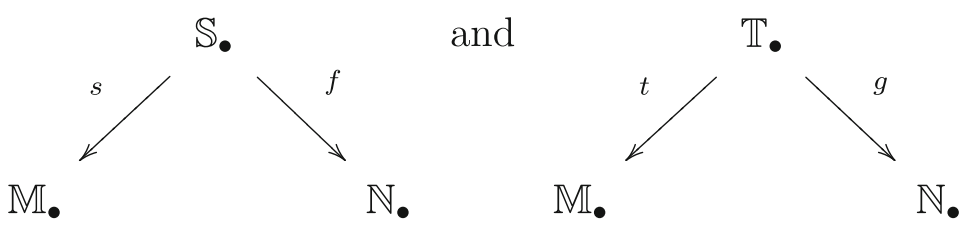

are equivalent in $\mathcal{D}_{k}^{-}(\mathbb{A}$-mod $)$, so injectivity is proved.

We obtain as a corollary Gerstenhaber and Schack's Invariance Theorem.

Corollary 4.10 (The Invariance Theorem) The natural transformation induced by $\mathbf{d}^{*}$ induces and isomorphism

$$
\mathbf{E x t}_{\mathbb{A}, k}^{\bullet}(-,-) \longrightarrow \mathbf{E x t}_{\mathbb{A}^{\prime}, k}^{\bullet}\left((-)^{\prime},(-)^{\prime}\right)
$$

Proof Theorem 4.6 implies that we have the isomorphisms

$$
\begin{aligned}
\operatorname{Ext}_{\mathbb{A}, \mathbf{k}}^{i}(\mathbb{M}, \mathbb{N}) & \simeq \operatorname{Mor}_{\mathcal{D}_{k}^{-}(\mathbb{A}-\mathbf{m o d})}\left(\mathbb{M}_{\bullet}, \mathbb{N}_{\bullet}[i]\right) \\
\mathbf{E x t}_{\mathbb{A}^{\prime}, \mathbf{k}}^{i}\left(\mathbb{M}^{\prime}, \mathbb{N}^{\prime}\right) & \simeq \operatorname{Mor}_{\mathcal{D}_{k}^{-}\left(\mathbb{A}^{\prime}-\mathbf{m o d}\right)}\left(\mathbb{M}_{\bullet}^{\prime}, \mathbb{N}_{\bullet}^{\prime}[i]\right)
\end{aligned}
$$

Since $\mathbf{d}^{*}$ is full and faithful we get the desired isomorphism.

\section{The general cohomology comparison theorem}

To each diagram of algebras $\mathbb{A}$ over a poset $\mathcal{C}$, Gerstenhaber and Schack associated a single algebra $\mathbb{A} !=$ of the row-finite $\mathcal{C} \times \mathcal{C}$ matrices $\left(a_{i j}\right)$ with $a_{i j} \in \mathbb{A}^{i}$ if $i \leq j$ and $a_{i j}=0$ otherwise. The addition is componentwise and the multiplication $\left(a_{i j}\right)\left(b_{i j}\right)=$ $\left(c_{i j}\right)$ is induced by the matrix multiplication with the understanding that, for $h \leq$ $i \leq j$, the summand $a_{h i} b_{i j}$ of $c_{h j}$ is regarded as $a_{h i} b_{i j}=a_{h i} \varphi^{h i}\left(b_{i j}\right)$. For our purpose it is convenient to use the equivalent representation $\mathbb{A} !=\prod_{i \in \mathcal{C}} \coprod_{i \leq j} \mathbb{A}^{i} \varphi^{i j}$, as $k$-bimodule. Here $\varphi^{i j}$ serve to distinguish distinct copies of $\mathbb{A}^{i}$ from one another. The general element of $\mathbb{A}^{i} \varphi^{i j}$ will be denoted $a^{i} \varphi^{i j}$. The multiplication is defined componentwise and subject to the rule: $\left(a^{h} \varphi^{h i}\right)\left(a^{j} \varphi^{j l}\right)=a^{h} \varphi^{h i}\left(a^{j}\right) \varphi^{h l}$ if $i=j$ and 0 otherwise.

Let $1_{i}$ the unit element of $\mathbb{A}^{i}$. Since $\left(a^{h} \varphi^{h i}\right)\left(1_{i} \varphi^{i j}\right)=a^{h} \varphi^{h j}$ and $\left(1_{i} \varphi^{h i}\right)\left(a^{i} \varphi^{i j}\right)=$ $\varphi^{h i}\left(a^{i}\right) \varphi^{h j}$ we may abbreviate $1_{i} \varphi^{i j}$ to $\varphi^{i j}$. The maps $\varphi^{i j}$ are then elements of $\mathbb{A}$ ! and $\varphi^{h i} \varphi^{i j}=\varphi^{h j} ; \varphi^{h i} \varphi^{j l}=0$ if $i \neq j$.

Gerstenhaber and Schack defined the functor

$$
!: \mathbb{A}^{e}-\bmod \longrightarrow(\mathbb{A} !)^{e}-\bmod , \text { such that } \mathbb{A} \longrightarrow \mathbb{A} !
$$

by setting for any $\mathbb{A}^{e}$-module $\mathbb{M}$,

$$
\mathbb{M} !=\prod_{i \in \mathcal{C}} \coprod_{i \leq j} \mathbb{M}^{i} \varphi^{i j} \text { as a } k \text {-bimodule } .
$$


The actions of $\mathbb{A}$ ! are defined by:

$$
\begin{aligned}
& \left(a^{h} \varphi^{h i}\right)\left(m^{i} \varphi^{i j}\right)=a^{h} T_{\mathbb{M}}^{h i}\left(m^{i}\right) \varphi^{h j} \\
& \left(m^{h} \varphi^{h i}\right)\left(a^{i} \varphi^{i j}\right)=m^{h} \varphi^{h i}\left(a^{i}\right) \varphi^{h j} \\
& \left(a^{h} \varphi^{h i}\right)\left(m^{j} \varphi^{j l}\right)=0=\left(m^{h} \varphi^{h i}\right)\left(a^{j} \varphi^{j l}\right), \text { if } i \neq j .
\end{aligned}
$$

For $\eta \in \operatorname{Hom}_{\mathbb{A}^{e}}(\mathbb{N}, \mathbb{M})$ define $\eta ! \in \operatorname{Hom}_{(\mathbb{A} !)^{e}}(\mathbb{N} !, \mathbb{M} !)$ by $\eta !\left(n^{i} \varphi^{i j}\right)=\eta^{i}\left(n^{i}\right) \varphi^{i j}$.

The functor ! is exact and $k$-linear so it induces a $k$-linear functor between the relative derived categories $\mathcal{D}_{k}^{-}\left(\mathbb{A}^{e}\right.$-mod $)$ and $\mathcal{D}_{k}^{-}\left((\mathbb{A} !)^{e}\right.$-mod $)$. In [7] we proved that the induced functor is full and faithful.

Theorem 5.1 The functor $\mathcal{D}_{k}^{-}\left(\mathbb{A}^{e}\right.$-mod $) \stackrel{!}{\longrightarrow} \mathcal{D}_{k}^{-}\left((\mathbb{A} !)^{e}\right.$-mod $)$ is full and faithful. That is,

$$
\operatorname{Mor}_{\mathcal{D}_{k}^{-}\left(\mathbb{A}^{e}-\text { mod }\right)}\left(\mathbb{M}_{\bullet}, \mathbb{N}_{\bullet}\right) \stackrel{!}{\longrightarrow} \operatorname{Mor}_{\mathcal{D}_{k}^{-}\left((\mathbb{A} !)^{e}-\text { mod }\right)}\left(\mathbb{M}_{\bullet} !, \mathbb{N}_{\bullet} !\right)
$$

is an isomorphism of $k$-modules for all $\mathbb{M}_{\bullet}, \mathbb{N}_{\bullet} \in \mathcal{D}_{k}^{-}\left(\mathbb{A}^{e}\right.$-mod $)$.

As a corollary we obtained the Special Cohomology Comparison Theorem due to Gerstenhaber and Schack (see [3]).

Corollary 5.2 (Special Cohomology Comparison Theorem) The functor! induces an isomorphism of relative Yoneda cohomologies

$$
\operatorname{Ext}_{\mathbb{A}^{e}, \mathbf{k}}^{\bullet}((-),(-)) \cong \operatorname{Ext}_{(\mathbb{A} !)^{e}, \mathbf{k}}^{\bullet}((-) !,(-) !) .
$$

In particular, we have an isomorphism of relative Hochschild cohomologies

$$
\mathbf{H}^{\bullet}(\mathbb{A},(-)) \cong \mathbf{H}^{\bullet}(\mathbb{A} !,(-) !)
$$

The operation of subdividing is compatible with taking opposites and tensor products of diagrams in the obvious way. This means that $\left(\mathbb{A}^{\prime}\right)^{e}=\left(\mathbb{A}^{e}\right)^{\prime}$, so Theorem 4.9 allows us to go from bimodules over $\mathbb{A}$ to bimodules over $\mathbb{A}^{\prime}$.

As we noted in Proposition 3.1, the second subdivision of a small category is always a poset. Therefore, by combining Theorems 4.9 and 5.1, we get the main result of this paper in the form of the following theorem.

Theorem 5.3 Let $\mathcal{C}$ be an arbitrary small category and $\mathbb{A}$ be a diagram over $\mathcal{C}$. Then, the functor

$$
\mathbb{M}_{\bullet} \longrightarrow\left(\mathbb{M}_{\bullet}^{\prime \prime}\right) !
$$

between the categories $\mathcal{D}_{k}^{-}\left(\mathbb{A}^{e}-\mathbf{m o d}\right)$ and $\mathcal{D}_{k}^{-}\left(\left(\mathbb{A}^{\prime \prime} !\right)^{e}\right.$-mod $)$ is full and faithful. That is, the natural map 


$$
\operatorname{Mor}_{\mathcal{D}_{k}^{-}\left(\mathbb{A}^{e}-\mathbf{m o d}\right)}\left(\mathbb{M}_{\bullet}, \mathbb{N}_{\bullet}\right) \stackrel{(-)^{\prime \prime} !}{\longrightarrow} \operatorname{Mor}_{\mathcal{D}_{k}^{-}\left(\left(\mathbb{A}^{\prime \prime} !\right)^{e}-\mathbf{m o d}\right)}\left((\mathbb{M})_{\bullet}^{\prime \prime} !,(\mathbb{N})_{\bullet}^{\prime \prime !}\right)
$$

is an isomorphism of $k$-modules for all $\mathbb{M}_{\bullet}, \mathbb{N}_{\bullet} \in \mathcal{D}_{k}^{-}\left(\mathbb{A}^{e}\right.$-mod $)$.

As a corollary we obtain the General Cohomology Comparison Theorem of Gerstenhaber and Schack.

Corollary 5.4 (General Cohomology Comparison Theorem) Let $\mathcal{C}$ be a small category and $\mathbb{A}$ be a diagram over $\mathcal{C}$. Then the functor

$$
\mathbb{M} \longrightarrow\left(\mathbb{M}^{\prime \prime}\right) !
$$

between the categories $\mathbb{A}^{e}-\bmod$ and $\left(\mathbb{A}^{\prime \prime}\right) !^{e}-\bmod$ is full and faithful. The induced map

$$
\mathbf{E x t}_{\mathbb{A}^{e}, \mathbf{k}}^{\bullet}((-),(-)) \longrightarrow \mathbf{E x t}_{\left(\mathbb{A}^{\prime \prime}\right) ! e, \mathbf{k},}^{\bullet}\left((-)^{\prime \prime} !,(-)^{\prime \prime} !\right),
$$

$[\mathcal{E}] \longrightarrow\left[\mathcal{E}^{\prime \prime} !\right]$ is an isomorphism. In particular, there is an isomorphism of Hochschild cohomologies

$$
\mathbf{H}^{\bullet}(\mathbb{A},(-)) \cong \mathbf{H}^{\bullet}\left(\left(\mathbb{A}^{\prime \prime}\right) !,(-)^{\prime \prime} !\right)
$$

\section{References}

1. Gelfand, S.I., Manin, Y.I.: Methods of Homological Algebra. Springer, Berlin (1996)

2. Gerstenhaber, M., Schack, S.D.: Algebraic cohomology and deformation theory. In: Deformation Theory of Algebras and Structures and Applications, pp. 11-264. Kluwer, Dordrecht (1988)

3. Gerstenhaber, M., Schack, S.D.: The cohomology of presheaves of algebras: presheaves over a partially ordered set. Trans. Am. Math. Soc. 310, 135-165 (1988)

4. Lowen, W., Van Den Bergh, M.: A hochschild cohomology comparison theorem for prestacks. Trans. Am. Math. Soc. 363, 969-986 (2011)

5. MacLane, S.: Homology. Spinger, Berlin (1967)

6. Stancu, A.A.: Hochschild cohomology and derived categories. SUNY at Buffalo Ph.D. thesis (2006)

7. Stancu, A.A.: On the cohomology comparison theorem. J. Homotopy Relat. Struct. 6(1), 39-63 (2011) 